\title{
Coefficient Estimates for a Certain General Subclass of Analytic and Bi-Univalent Functions
}

\author{
Nanjundan Magesh ${ }^{1}$, Jagadeesan Yamini ${ }^{2}$ \\ ${ }^{1}$ Post-Graduate and Research Department of Mathematics, Government Arts College for Men, Krishnagiri, \\ India \\ ${ }^{2}$ Department of Mathematics, Govt First Grade College, Bangalore, India \\ Email: nmagi 2000@yahoo.co.in, yaminibalaji@gmail.com
}

Received 13 January 2014; revised 13 February 2014; accepted 20 February 2014

Copyright (C) 2014 by authors and Scientific Research Publishing Inc.

This work is licensed under the Creative Commons Attribution International License (CC BY).

http://creativecommons.org/licenses/by/4.0/

(c) (i) Open Access

\begin{abstract}
Motivated and stimulated especially by the work of Xu et al. [1], in this paper, we introduce and discuss an interesting subclass $\mathcal{G}_{\Sigma}^{\varphi, \psi}(\lambda)$ of analytic and bi-univalent functions defined in the open unit disc $\mathbb{U}$. Further, we find estimates on the coefficients $\left|a_{2}\right|$ and $\left|a_{3}\right|$ for functions in this subclass. Many relevant connections with known or new results are pointed out.
\end{abstract}

\section{Keywords}

\section{Analytic Functions, Univalent Functions, Bi-Univalent Functions, Bi-Starlike Functions}

\section{Introduction}

Let $\mathcal{A}$ denote the class of functions of the form

$$
f(z)=z+\sum_{n=2}^{\infty} a_{n} z^{n}
$$

which are analytic in the open unit disc $\mathbb{U}=\{z: z \in \mathbb{C}$ and $|z|<1\}$. Further, by $\mathcal{S}$ we shall denote the class of all functions in $\mathcal{A}$ which are univalent in $\mathbb{U}$. Some of the important and well-investigated subclasses of the univalent function class $\mathcal{S}$ include (for example) the class $\mathcal{S}^{*}(\beta)$ of starlike functions of order $\beta$ $(0 \leq \beta<1)$ in $\mathbb{U}$ and the class $\mathcal{S S}^{*}(\alpha)$ of strongly starlike functions of order $\alpha \quad(0<\alpha \leq 1)$ in $\mathbb{U}$. It is well known that every function $f \in \mathcal{S}$ has an inverse $f^{-1}$, defined by 


$$
f^{-1}(f(z))=z \quad(z \in \mathbb{U})
$$

and

$$
f\left(f^{-1}(w)\right)=w \quad\left(|w|<r_{0}(f) ; r_{0}(f) \geq \frac{1}{4}\right)
$$

where

$$
f^{-1}(w)=w-a_{2} w^{2}+\left(2 a_{2}^{2}-a_{3}\right) w^{3}-\left(5 a_{2}^{3}-5 a_{2} a_{3}+a_{4}\right) w^{4}+\cdots
$$

A function $f \in \mathcal{A}$ is said to be bi-univalent in $\mathbb{U}$ if both $f$ and $f^{-1}$ are univalent in $\mathbb{U}$. We denote by $\Sigma$ the class of all bi-univalent functions in $\mathbb{U}$. For a brief history and interesting examples of functions in the class $\Sigma$ see [2] and the references therein.

In fact, the study of the coefficient problems involving bi-univalent functions was revived recently by Srivastava et al. [2]. Various subclasses of the bi-univalent function class $\Sigma$ were introduced and non-sharp estimates on the first two Taylor-Maclaurin coefficients $\left|a_{2}\right|$ and $\left|a_{3}\right|$ of functions in these subclasses were found in several recent investigations (see, for example, [3]-[13]). The aforecited all these papers on the subject were motivated by the pioneering work of Srivastava et al. [2]. But the coefficient problem for each of the following Taylor-Maclaurin coefficients $\left|a_{n}\right|(n \in \mathbb{N} \backslash\{1,2\} ; \mathbb{N}:=\{1,2,3, \cdots\})$ is still an open problem.

Motivated by the aforecited works (especially [1]), we introduce the following subclass $\mathcal{G}_{\Sigma}^{\varphi, \psi}(\lambda)$ of the analytic function class $\mathcal{A}$.

Definition 1 Let $f \in \mathcal{A}$ and the functions $\varphi, \psi: \mathbb{U} \rightarrow \mathbb{C}$ be so constrained that $\min \{\mathfrak{R}(\varphi(z)), \mathfrak{R}(\psi(z))\}>0, \quad z \in \mathbb{U}$ and $\varphi(0)=\psi(0)=1$. We say that $f \in \mathcal{G}_{\Sigma}^{\varphi, \psi}(\lambda)$ if the following conditions are satisfied: $f \in \Sigma$,

$$
\frac{z f^{\prime}(z)}{(1-\lambda) f(z)+\lambda z f^{\prime}(z)} \in \varphi(\mathbb{U}) \quad(0 \leq \lambda<1 ; z \in \mathbb{U})
$$

and

$$
\frac{w g^{\prime}(w)}{(1-\lambda) g(w)+\lambda w g^{\prime}(w)} \in \psi(\mathbb{U}) \quad(0 \leq \lambda<1 ; w \in \mathbb{U}),
$$

where the function $g$ is the extension of $f^{-1}$ to $\mathbb{U}$.

We note that, for the different choices of the functions $\varphi$ and $\psi$, we get interesting known and new subclasses of the analytic function class $\mathcal{A}$. For example, if we set

$$
\varphi(z)=\left(\frac{1+z}{1-z}\right)^{\alpha} \text { and } \psi(z)=\left(\frac{1-z}{1+z}\right)^{\alpha} \quad(0<\alpha \leq 1 ; z \in \mathbb{U}),
$$

in the class $\mathcal{G}_{\Sigma}^{\varphi, \psi}(\lambda)$ then we have $\mathcal{S S}_{\Sigma}^{*}(\alpha, \lambda)$. Also, $f \in \mathcal{S S}_{\Sigma}^{*}(\alpha, \lambda)$ if the following conditions are satisfied:

$$
f \in \Sigma, \quad \arg \left(\frac{z f^{\prime}(z)}{(1-\lambda) f(z)+\lambda z f^{\prime}(z)}\right) \mid<\frac{\alpha \pi}{2} \quad(0<\alpha \leq 1 ; 0 \leq \lambda<1 ; z \in \mathbb{U})
$$

and

$$
\left|\arg \left(\frac{w g^{\prime}(w)}{(1-\lambda) g(w)+\lambda w g^{\prime}(w)}\right)\right|<\frac{\alpha \pi}{2} \quad(0<\alpha \leq 1 ; 0 \leq \lambda<1 ; w \in \mathbb{U}),
$$

where $g$ is the extension of $f^{-1}$ to $\mathbb{U}$.

Similarly, if we let

$$
\varphi(z)=\frac{1+(1-2 \beta) z}{1-z} \text { and } \psi(z)=\frac{1-(1-2 \beta) z}{1+z} \quad(0 \leq \beta<1 ; z \in \mathbb{U}),
$$

in the class $\mathcal{G}_{\Sigma}^{\varphi, \psi}(\lambda)$ then we get $\mathcal{S}_{\Sigma}^{*}(\beta, \lambda)$. Further, we say that $f \in \mathcal{S}_{\Sigma}^{*}(\beta, \lambda)$ if the following conditions 
are satisfied:

$$
f \in \Sigma, \quad \Re\left(\frac{z f^{\prime}(z)}{(1-\lambda) f(z)+\lambda z f^{\prime}(z)}\right)>\beta \quad(0 \leq \beta<1 ; 0 \leq \lambda<1 ; z \in \mathbb{U})
$$

and

$$
\Re\left(\frac{w g^{\prime}(w)}{(1-\lambda) g(w)+\lambda w g^{\prime}(w)}\right)>\beta \quad(0 \leq \beta<1 ; 0 \leq \lambda<1 ; w \in \mathbb{U}),
$$

where $g$ is the extension of $f^{-1}$ to $\mathbb{U}$.

The classes $\mathcal{S S}_{\Sigma}^{*}(\alpha, \lambda)$ and $\mathcal{S}_{\Sigma}^{*}(\beta, \lambda)$ were introduced and studied by Murugusundaramoorthy et al. [12], Definition 1.1 and Definition 1.2]. The classes $\mathcal{S S}_{\Sigma}^{*}(\alpha, 0):=\mathcal{S S}_{\Sigma}^{*}(\alpha)$ and $\mathcal{S}_{\Sigma}^{*}(\beta, 0):=\mathcal{S}_{\Sigma}^{*}(\beta)$ are strongly bi-starlike functions of order $\alpha$ and bi-starlike functions of order $\beta$ respectively. The classes $\mathcal{S S}_{\Sigma}^{*}(\alpha)$ and $\mathcal{S}_{\Sigma}^{*}(\beta)$ were introduced and studied by Brannan and Taha [14], Definition 1.1 and Definition 1.2]. In addition, we note that, $\mathcal{G}_{\Sigma}^{\varphi, \psi}(0):=\mathcal{B}_{\Sigma}^{\varphi, \psi}$ was introduced and studied by Bulut [4], Definition 3].

Motivated and stimulated by Bulut [4] and Xu et al. [1] (also [10]), in this paper, we introduce a new subclass $\mathcal{G}_{\Sigma}^{\varphi, \psi}(\lambda)$ and obtain the estimates on the coefficients $\left|a_{2}\right|$ and $\left|a_{3}\right|$ for functions in aforementioned class, employing the techniques used earlier by Xu et al. [1].

\section{A Set of General Coefficient Estimates}

In this section we state and prove our general results involving the bi-univalent function class $\mathcal{G}_{\Sigma}^{\varphi, \psi}(\lambda)$ given by Definition 1.

Theorem 1 Let $f(z)$ be of the form (1.1). If $f \in \mathcal{G}_{\Sigma}^{\varphi, \psi}(\lambda)$, then

$$
\left|a_{2}\right| \leq \min \left\{\sqrt{\frac{\left|\varphi^{\prime}(0)\right|^{2}+\left|\psi^{\prime}(0)\right|^{2}}{2(1-\lambda)^{2}}}, \frac{\sqrt{\left|\varphi^{\prime \prime}(0)\right|+\left|\psi^{\prime \prime}(0)\right|}}{2(1-\lambda)}\right\}
$$

and

$$
\left|a_{3}\right| \leq \min \left\{\frac{\left|\varphi^{\prime}(0)\right|^{2}+\left|\psi^{\prime}(0)\right|^{2}}{2(1-\lambda)^{2}}+\frac{\left|\varphi^{\prime \prime}(0)\right|+\left|\psi^{\prime \prime}(0)\right|}{8(1-\lambda)}, \frac{(3-\lambda)\left|\varphi^{\prime \prime}(0)\right|+(1+\lambda)\left|\psi^{\prime \prime}(0)\right|}{8(1-\lambda)^{2}}\right\} .
$$

Proof 1 Since $f \in \mathcal{G}_{\Sigma}^{\varphi, \psi}(\lambda)$. From (1.3) and (1.4), we have,

$$
\frac{z f^{\prime}(z)}{(1-\lambda) f(z)+\lambda z f^{\prime}(z)}=\varphi(z) \quad(z \in \mathbb{U})
$$

and

$$
\frac{w g^{\prime}(w)}{(1-\lambda) g(w)+\lambda w g^{\prime}(w)}=\psi(w) \quad(w \in \mathbb{U}),
$$

where

$$
\varphi(z)=1+\varphi_{1} z+\varphi_{2} z^{2}+\cdots
$$

and

$$
\psi(z)=1+\psi_{1} z+\psi_{2} z^{2}+\cdots
$$

satisfy the conditions of Definition 1. Now, upon equating the coefficients of $\frac{z f^{\prime}(z)}{(1-\lambda) f(z)+\lambda z f^{\prime}(z)}$ with those of $\varphi(z)$ and the coefficients of $\frac{w g^{\prime}(w)}{(1-\lambda) g(w)+\lambda w g^{\prime}(w)}$ with those of $\psi(w)$, we get 


$$
\begin{aligned}
& (1-\lambda) a_{2}=\varphi_{1} \\
& \left(\lambda^{2}-1\right) a_{2}^{2}+2(1-\lambda) a_{3}=\varphi_{2} \\
- & (1-\lambda) a_{2}=\psi_{1}
\end{aligned}
$$

and

$$
\left(\lambda^{2}-4 \lambda+3\right) a_{2}^{2}-2(1-\lambda) a_{3}=\psi_{2} .
$$

From (1.7) and (1.9), we get

$$
\varphi_{1}=-\psi_{1}
$$

and

$$
2(1-\lambda)^{2} a_{2}^{2}=\varphi_{1}^{2}+\psi_{1}^{2}
$$

From (1.8) and (1.10), we obtain

$$
2(1-\lambda)^{2} a_{2}^{2}=\varphi_{2}+\psi_{2} .
$$

Therefore, we find from (1.12) and (1.13) that

and

$$
a_{2}^{2}=\frac{\varphi_{1}^{2}+\psi_{1}^{2}}{2(1-\lambda)^{2}} \text {. }
$$

$$
a_{2}^{2}=\frac{\varphi_{2}+\psi_{2}}{2(1-\lambda)^{2}} .
$$

Since $\varphi(z) \in \varphi(\mathbb{U})$ and $\psi(z) \in \psi(\mathbb{U})$, we immediately have

$$
\left|a_{2}\right|^{2} \leq \frac{\left|\varphi^{\prime}(0)\right|^{2}+\left|\psi^{\prime}(0)\right|^{2}}{2(1-\lambda)^{2}}
$$

and

$$
\left|a_{2}\right|^{2} \leq \frac{\left|\varphi^{\prime \prime}(0)\right|^{2}+\left|\psi^{\prime \prime}(0)\right|^{2}}{4(1-\lambda)^{2}}
$$

respectively. So we get the desired estimate on $\left|a_{2}\right|$ as asserted in (1.5).

Next, in order to find the bound on $\left|a_{3}\right|$, by subtracting (1.10) from (1.8), we get

$$
4(1-\lambda) a_{3}-4(1-\lambda) a_{2}^{2}=\varphi_{2}-\psi_{2} \text {. }
$$

Upon substituting the values of $a_{2}^{2}$ from (1.14) and (1.15) into (1.16), we have

$$
a_{3}=\frac{\varphi_{1}^{2}+\psi_{1}^{2}}{2(1-\lambda)^{2}}+\frac{\varphi_{2}-\psi_{2}}{4(1-\lambda)}
$$

and

$$
a_{3}=\frac{(3-\lambda) \varphi_{2}+(1+\lambda) \psi_{2}}{4(1-\lambda)^{2}}
$$

respectively. Since $\varphi(z) \in \varphi(\mathbb{U})$ and $\psi(z) \in \psi(\mathbb{U})$, we readily get

$$
\left|a_{3}\right| \leq \frac{\left|\varphi^{\prime}(0)\right|^{2}+\left|\psi^{\prime}(0)\right|^{2}}{2(1-\lambda)^{2}}+\frac{\left|\varphi^{\prime \prime}(0)\right|+\left|\psi^{\prime \prime}(0)\right|}{8(1-\lambda)},
$$

and 


$$
\left|a_{3}\right| \leq \frac{(3-\lambda)\left|\varphi^{\prime \prime}(0)\right|+(1+\lambda)\left|\psi^{\prime \prime}(0)\right|}{8(1-\lambda)^{2}} .
$$

This completes the proof of Theorem 1.

If we choose

$$
\varphi(z)=\left(\frac{1+z}{1-z}\right)^{\alpha} \text { and } \psi(z)=\left(\frac{1-z}{1+z}\right)^{\alpha} \quad(0<\alpha \leq 1, z \in \mathbb{U})
$$

in Theorem 1, we have the following corollary.

Corollary 1 Let $f(z)$ be of the form (1.1) and in the class $\mathcal{S S}_{\Sigma}^{*}(\alpha, \lambda)$. Then

$$
\left|a_{2}\right| \leq \min \left\{\frac{2 \alpha}{1-\lambda}, \frac{\sqrt{2} \alpha}{1-\lambda}\right\}
$$

and

$$
\left|a_{3}\right| \leq \min \left\{\frac{4 \alpha^{2}}{(1-\lambda)^{2}}+\frac{\alpha^{2}}{1-\lambda}, \frac{2 \alpha^{2}}{(1-\lambda)^{2}}\right\} .
$$

If we set

$$
\varphi(z)=\frac{1+(1-2 \beta) z}{1-z} \text { and } \psi(z)=\frac{1-(1-2 \beta) z}{1+z} \quad(0 \leq \beta<1, z \in \mathbb{U})
$$

in Theorem 1, we readily have the following corollary.

Corollary 2 Let $f(z)$ be of the form (1.1) and in the class $\mathcal{S}_{\Sigma}^{*}(\beta, \lambda)$. Then

$$
\left|a_{2}\right| \leq \min \left\{\frac{2(1-\beta)}{1-\lambda}, \frac{\sqrt{2(1-\beta)}}{1-\lambda}\right\}
$$

and

$$
\left|a_{3}\right| \leq \min \left\{\frac{4(1-\beta)^{2}}{(1-\lambda)^{2}}+\frac{1-\beta}{1-\lambda}, \frac{2(1-\beta)}{(1-\lambda)^{2}}\right\} .
$$

Remark 1 The estimates on the coefficients $\left|a_{2}\right|$ and $\left|a_{3}\right|$ of Corollaries 1 and 2 are improvement of the estimates obtained in [10], Theorems 4 and 5]. Taking $\lambda=0$ in Corollaries 1 and 2, the estimates on the coefficients $\left|a_{2}\right|$ and $\left|a_{3}\right|$ are improvement of the estimates in [14], Theorems 2.1 and 4.1]. When $\lambda=0$ the results discussed in this article reduce to results in [4]. Similarly, various other interesting corollaries and consequences of our main result can be derived by choosing different $\varphi$ and $\psi$.

\section{Acknowledgements}

The authors would like to record their sincere thanks to the referees for their valuable suggestions.

\section{Funding}

The work is supported by UGC, under the grant F.MRP-3977/11 (MRP/UGC-SERO) of the first author.

\section{References}

[1] Xu, Q.-H., Gui, Y.-C. and Srivastava, H.M. (2012) Coefficient Estimates for a Certain Subclass of Analytic and Bi-Univalent Functions. Applied Mathematics Letters, 25, 990-994.

[2] Srivastava, H.M., Mishra, A.K. and Gochhayat, P. (2010) Certain Subclasses of Analytic and Bi-Univalent Functions. Applied Mathematics Letters, 23, 1188-1192.

[3] Ali, R.M., Lee, S.K., Ravichandran, V. and Supramanian, S. (2012) Coefficient Estimates for Bi-Univalent Ma-Minda Starlike and Convex Functions. Applied Mathematics Letters, 25, 344-351. 
[4] Bulut, S. (2013) Coefficient Estimates for a Class of Analytic and Bi-Univalent Functions. Novi Sad Journal of Mathematics, 43, 59-65.

[5] Çağlar, M., Orhan, H. and Yağmur, N. (2012) Coefficient Bounds for New Subclasses of Bi-Univalent Functions. FILOMAT, 27, 1165-1171.

[6] Frasin, B.A. and Aouf, M.K. (2011) New Subclasses of Bi-Univalent Functions. Applied Mathematics Letters, 24, 1569-1573.

[7] Hayami, T. and Owa, S. (2012) Coefficient Bounds for Bi-Univalent Functions. Pan American Mathematical Journal, 22, 15-26.

[8] Li, X.-F. and Wang, A.-P. (2012) Two New Subclasses of Bi-Univalent Functions. International Mathematical Forum, 7, 1495-1504.

[9] Magesh, N., Rosy, T. and Varma, S. (2013) Coefficient Estimate Problem for a New Subclass of Biunivalent Functions. Journal of Complex Analysis, 2013, Article ID: 474231, 3 Pages.

[10] Murugusundaramoorthy, G., Magesh, N. and Prameela, V. (2013) Coefficient Bounds for Certain Subclasses of Bi-Univalent Functions. Abstract and Applied Analysis, 2013, Article ID: 573017, 3 Pages.

[11] Srivastava, H.M., Bulut, S., Çağlar, M. and Yağmur, N. (2013) Coefficient Estimates for a General Subclass of Analytic and Bi-Univalent Functions. FILOMAT, 27, 831-842.

[12] Srivastava, H.M., Murugusundaramoorthy, G. and Magesh, N. (2013) On Certain Subclasses of Bi-Univalent Functions Associated with Hohlov Operator. Global J. Math. Anal, 1, 67-73.

[13] Xu, Q.-H., Xiao, H.-G. and Srivastava, H. M. (2012) A Certain General Subclass of Analytic and Bi-Univalent Functions and Associated Coefficient Estimate Problems. Applied Mathematics and Computation, 218, 11461-11465.

[14] Brannan, D.A. and Taha, T.S. (1986) On Some Classes of Bi-Univalent Functions. Studia Universitatis Babeş-Bolyai. Mathematica, 31, 70-77. 